\title{
Serial analysis of 3D H-1 MRSI for patients with newly diagnosed GBM treated with combination therapy that includes bevacizumab
}

\author{
Sarah J. Nelson ${ }^{1,3,4,5} \cdot$ Yan Li $^{1} \cdot$ Janine M. Lupo ${ }^{1}$ Marram Olson ${ }^{1} \cdot$ \\ Jason C. Crane ${ }^{1} \cdot$ Annette Molinaro $^{2,3} \cdot$ Ritu Roy $^{3} \cdot$ Jennifer Clarke ${ }^{2,3,4}$. \\ Nicholas Butowski ${ }^{2,3} \cdot$ Michael Prados $^{2,3} \cdot$ Soonmee Cha ${ }^{1} \cdot$ Susan M. Chang ${ }^{2,3}$ (D)
}

Received: 27 February 2016 / Accepted: 31 July 2016 / Published online: 17 August 2016

(C) The Author(s) 2016. This article is published with open access at Springerlink.com

\begin{abstract}
Interpretation of changes in the T1- and T2-weighted MR images from patients with newly diagnosed glioblastoma (GBM) treated with standard of care in conjunction with anti-angiogenic agents is complicated by pseudoprogression and pseudoresponse. The hypothesis being tested in this study was that 3D H-1 magnetic resonance spectroscopic imaging (MRSI) provides estimates of levels of choline, creatine, $\mathrm{N}$-acetylaspartate (NAA), lactate and lipid that change in response to treatment and that metrics describing these characteristics are associated with survival. Thirty-one patients with newly diagnosed GBM and being treated with radiation therapy (RT), temozolomide, erlotinib and bevacizumab were recruited to receive serial MR scans that included 3-D lactate edited MRSI at baseline, mid-RT, post-RT and at specific follow-up time points. The data were processed to provide estimates of metrics representing changes in metabolite levels relative to normal appearing brain. Cox proportional hazards analysis was applied to examine the relationship of these parameters with progression free
\end{abstract}

Sarah J. Nelson

sarah.nelson@ucsf.edu

1 Department of Radiology and Biomedical Imaging, University of California, San Francisco, CA, USA

2 Department of Neurological Surgery, University of California, San Francisco, CA, USA

3 Helen Diller Family Comprehensive Cancer Center, University of California, San Francisco, CA, USA

4 Department of Neurology, University of California, San Francisco, CA, USA

5 Department of Bioengineering and Therapeutic Sciences, University of California, San Francisco, CA, USA survival (PFS) and overall survival (OS). There were significant reductions in parameters that describe relative levels of choline to NAA and creatine, indicating that the treatment caused a decrease in tumor cellularity. Changes in the levels of lactate and lipid relative to the NAA from contralateral brain were consistent with vascular normalization. Metabolic parameters from the first serial followup scan were associated with PFS and OS, when accounting for age and extent of resection. Integrating metabolic parameters into the assessment of patients with newly diagnosed GBM receiving therapies that include antiangiogenic agents may be helpful for tracking changes in tumor burden, resolving ambiguities in anatomic images caused by non-specific treatment effects and for predicting outcome.

Keywords Metabolic imaging · MRSI ·

Newly diagnosed glioblastoma .

Anti-angiogenic therapy $\cdot$ Survival

\section{Introduction}

The current standard of care for patients with newly diagnosed glioblastoma (GBM) is maximal safe resection, followed by radiation therapy and temozolomide [1]. A variety of investigational agents are being considered in conjunction with this strategy in order to improve outcome. Evaluation of combination therapies is complicated by differences in their mechanisms of action that lead to difficulties in assessing the spatial extent of tumor and ambiguities in the interpretation of conventional anatomic images. One such effect that has been reported to occur in up to $30 \%$ of patients treated with radiation and temozolomide is pseudoprogression, which is characterized by an increase in the size of 
the enhancing lesion that subsequently disappears without further intervention [2].

Given that GBM are characterized by abnormal vasculature, there has been great interest in using treatment regimens that include anti-angiogenic agents [3-5]. The dramatic reduction in the size of the contrast enhancing (CE) lesion that was observed following treatment of recurrent GBM with bevacizumab was very encouraging and led to conditional approval for its use. Subsequent studies in populations of patients with newly diagnosed GBM have shown longer progression free survival but have not established a significant improvement in overall survival $[6,7]$. For cases where the treatment regime includes such antiangiogenic agents, it is not clear whether early reductions in the CE lesion are indicative of successful treatment or if they are associated with pseudo- or true response.

The ambiguities in interpreting changes in the size of the $\mathrm{CE}$ lesion have highlighted the need to develop alternative approaches for assessing treatment response. The RANO criteria are a first step in that direction [8], as they consider changes in the cross sectional diameter of both the CE and $\mathrm{T} 2$ lesion. A further suggestion has been that estimating the three dimensional (3D) volumes of the anatomic would provide a more precise assessment of their absolute and relative changes with time. While providing more precise quantification of lesion size is definitely of interest, it is not clear whether this would be able to address pseudoprogression and pseudoresponse. Having an imaging modality that makes it possible to track biological response to therapy is likely to be a more promising approach. Previous studies that applied H-1 MR spectroscopic imaging (MRSI) to evaluate patients with GBM have shown that the magnitude and spatial extent of regions with abnormal metabolism is often quite different from areas corresponding to the $\mathrm{CE}$ and $\mathrm{T} 2$ lesions [9-12]. This suggests that metabolic imaging can provide complementary information about changes in tumor burden that would be helpful in assessing response to therapy.

The purpose of this study was to obtain 3D H-1 MRSI data from a cohort of patients with newly diagnosed GBM being treated with radiation therapy, temozolomide, erlotinib and bevacizumab in order to evaluate treatment induced changes in the metabolic characteristics of the lesion. The hypothesis being tested was that levels of choline, creatine, $N$-acetylaspartate (NAA), lactate and lipid change in response to treatment and that metrics describing these characteristics are associated with survival.

\section{Methods}

Thirty-one patients with pathologically confirmed newly diagnosed GBM, who were being treated as part of a single institution Phase II clinical trial [13] were recruited for this study. External beam radiation therapy (RT) was initiated within 5 weeks of diagnosis and delivered an average dose of 60 Gy to the tumor site in 2-Gy fractions over a 6-week period. Temozolomide was given concurrently and after RT at a daily dose of $75 \mathrm{mg} / \mathrm{m}^{2}$. Patients who were not on anti-epileptic drugs received $150 \mathrm{mg} /$ day of erlotinib and patients who were on anti-epileptic drugs received $500 \mathrm{mg} /$ day starting on day 1 of RT. Bevacizumab was prescribed at a dose of $10 \mathrm{mg} / \mathrm{kg}$ IV every 14 days starting in week 2 of RT. All patients participating in the study gave informed consent, according to the guidelines of our institutional review board. Overall survival (OS) was calculated as the time to death from the start of treatment and progression free survival (PFS) as the time from the start of treatment to the examination at which the patient was determined to have progressed based upon the RANO criteria or the time of death if there had been no prior progression [8].

\section{Imaging protocol}

Patients received MR exams at multiple time points, including following surgical resection but prior to RT (pre-RT), between 3 and 5 weeks into treatment (mid-RT), within 2 weeks after completion of RT (post-RT), and at the next follow-up scan that was approximately 4 months after the baseline time point (Fup1). All scans were obtained with a $3 \mathrm{~T} \mathrm{MR}$ scanner (GE Healthcare, Milwaukee, WI), the body coil for transmission and an 8-channel phased array coil for reception. Standard anatomical imaging comprised axial T2-weighted fluid attenuated inversion recovery (FLAIR) images (TR/ $\mathrm{TE} / \mathrm{TI}=9500 / 122 / 2375 \mathrm{~ms}$, matrix $=256 \times 256$, slice thickness $=3 \mathrm{~mm}, \mathrm{FOV}=24 \times 24 \mathrm{~cm}$ ), and pre- and post-contrast volumetric $\mathrm{T} 1$-weighted inversion recovery spoiled gradient echo images $(\mathrm{TR} / \mathrm{TE}=8.86 / 2.50 \mathrm{~ms}$, matrix $=256 \times 256$, slice thickness $=1.5 \mathrm{~mm}, \mathrm{FOV}=24 \times 24 \mathrm{~cm}, \mathrm{TI}=400 \mathrm{~ms}$, Flip angle $=15^{\circ}$ ).

Lactate edited 3D H-1 MRSI data [14] were obtained with CHESS water suppression, very selective saturation (VSS) outer volume suppression and PRESS volume selection $(\mathrm{TR}=1104$ or $1300 \mathrm{~ms}, \mathrm{TE}=144 \mathrm{~ms}$, acquisition matrix of $16 \times 16 \times 16$ or $18 \times 18 \times 16$, nominal spatial resolution of $1 \mathrm{~cm}^{3}$ ). Phase encoding was applied in the right-left and anterior-posterior directions with fly-back trajectories in the superior-inferior direction. An over-PRESS factor of 1.5 was applied with VSS bands to avoid chemical shift artifacts and to suppress residual lipid signals. The total acquisition time was $9.5 \mathrm{~min}$, with 712 complex spectral points read indirectly using the EPI train of gradient pulses and spectral bandwidth of $988 \mathrm{~Hz}$. The PRESS selected volume was defined using the anatomic images and generally covered the lesion and 200-500 cc of normal tissue. Areas with sharply varying magnetic susceptibility and lipid contamination were avoided whenever possible. 


\section{Processing MRI and MRSI data}

After each examination, the DICOM images and raw spectral data were transferred offline for post-processing. The volumetric post-contrast $\mathrm{T} 1$ weighted image was used as a reference and the other images were aligned to the same coordinate system through rigid body transformations that maximized the normalized mutual information $[2,15]$. The $\mathrm{CE}$ lesion was manually defined on the post-contrast $\mathrm{T} 1$ weighted images. Any enhancement that was also present on the pre-contrast T1 images was assumed to represent acute blood products and was excluded. The T2 lesion was segmented based on the region of hyperintensity on FLAIR images. The resection cavity was excluded from all ROIs. T1 subtraction images [16] were generated from the pre- and post-contrast T1-weighted images and the T1s lesion was identified by thresholding the region of hyperintensity within the $\mathrm{T} 2$ region. Normal appearing brain was identified by brain extraction, followed by removal of regions within the $\mathrm{T} 2$ lesion ROI.

The spectral data were processed as described previously $[17,18]$ to provide levels of choline, creatine, NAA, lactate and lipid. The choline-NAA index (CNI) is a metric developed to describe the deviation of choline and NAA in regions with normal metabolite levels in the same individual $[19,20]$. The calculation is performed using an automated, iterative procedure that uses choline and NAA levels in the spectral array from each examination separately and is independent of the anatomic images. Once the CNI map had been generated for each patient and time point, their metabolic lesion was defined as the region having values greater than or equal to 2 (CNI2). Figure 1 shows an example of the spectral data and CNI overlays from the Fup1 scan for a patient with a relatively large metabolic lesion at this time point. The CNI intensities were interpolated to the resolution of the anatomic images for display purposes and the overlay was thresholded to show only values above 2 in order to highlight the spatial extent of the metabolic lesion. The choline to creatine index (CCrI) was estimated in a similar manner, but with the definition of abnormal voxels being based upon those that had been identified during the CNI calculation.

To be able to compare across serial exams, levels of choline, creatine, and NAA intensities were normalized by their median value in voxels from normal appearing brain (nCho,

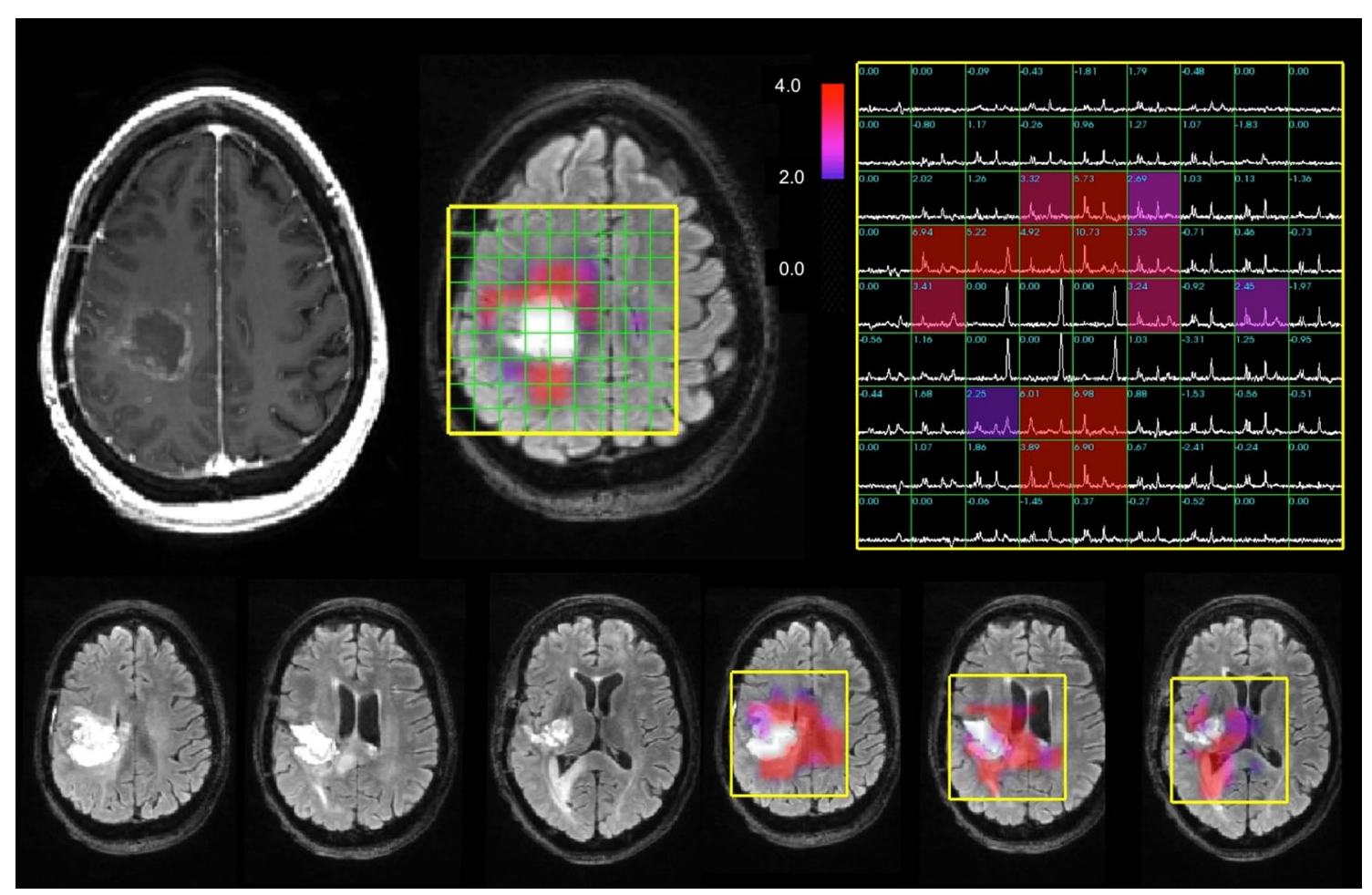

Fig. 1 Anatomic images, spectra and CNI color overlays from the Fup1 scan for a 47 year old female patient with a KPS of 90, sub-total resection, a low PFS of 159 days and a low OS of 206 days. The CNI maps were thresholded to show only values that were 2 or higher in order to highlight the metabolic lesion. The median and maximum CNI were 4.8 and 19.9. Note the high lipid peaks in the cavity, which are surrounded by areas with increased choline and decreased NAA that corresponded to the metabolic lesion. The residual CE volume was $3 \mathrm{~cm}^{3}$ is minimal and corresponds mainly to a thin rim around the resection. The volume of the T2 lesion at this time point is $29 \mathrm{~cm}^{3}$. As is shown from the FLAIR images and CNI overlays from the three lower slices, the metabolic lesion is relatively large and gives a clear picture of regions that are likely to reflect recurrent/residual tumor 
$\mathrm{nCr}$ and nNAA). Signals from lipid and lactate peaks were normalized with the median NAA from voxels in normal appearing brain (nLac and nLip). Parameters considered for analysis were the volume of the CNI2 and the median, maximum and summation of voxel values for the CNI, CCrI, $n L a c$ and nLip from within the CNI2 regions at each of the four time points.

\section{Statistical analysis}

Serial changes in imaging parameters were evaluated using Wilcoxon signed rank sign tests for paired analysis. Association with PFS and OS was assessed using Cox proportional hazards analysis, taking into account patient age and extent of resection. The cut-off for defining a significant result in this exploratory study was a $\mathrm{P}$ value of 0.05 .

\section{Results}

The median PFS for these patients was 404 days with 27 events and 4 subjects censored. The median OS was 603 days with 23 events and 8 subjects censored. There were 16 females and 15 males: median age was 52 years with a range of 21 to 75 , median KPS was 90 with a range of 60-90. There were 9 patients who were designated based upon clinical criteria as having received a gross total resection, 17 who had a sub-total resection and 5 who were characterized as having a biopsy.

\section{Changes in anatomic lesion volumes}

For the 22 patients who had anatomic scans at all 4 time points there were overall reductions in the volumes of the anatomic lesions with treatment. The mean pre-RT T2 lesion volume was $36.2 \mathrm{~cm}^{3}$, which subsequently decreased to $28.9 \mathrm{~cm}^{3}$ at mid-RT $(p>0.05)$, to $15.3 \mathrm{~cm}^{3}$ at post-RT $(\mathrm{p}<0.0001)$ and to $14.7 \mathrm{~cm}^{3}$ at Fup1 $(\mathrm{p}=0.0002)$. The mean CE lesion volume decreased from $5.2 \mathrm{~cm}^{3}$ at pre-RT to $3.0 \mathrm{~cm}^{3}$ at mid-RT $(\mathrm{p}=0.018), 2.1 \mathrm{~cm}^{3}$ at post-RT $(\mathrm{p}=0.0002)$ and $0.7 \mathrm{~cm}^{3}$ at Fup1 $(\mathrm{p}<0.0001)$. The mean T1s volume was obtained by thresholding the T1 subtraction image and showed similar changes to the CE lesion volumes but was overall larger in size at all four time points (mean volumes of 9.4, 5.8, 3.3 and $1.7 \mathrm{~cm}^{3}$ respectively). These results are represented in the bar graphs in Fig. 2.

\section{Changes in metabolic parameters}

At pre-RT, the CNI2 lesion volumes were much larger than the CE and T1s lesions (mean of 25.2 vs. 5.2 and $9.1 \mathrm{~cm}^{3}$ respectively). Although many of the $\mathrm{T} 2$ lesion volumes at pre-RT were larger than the CNI2 lesions, the mean overlap was only $11.2 \mathrm{~cm}^{3}$. This indicates that there were subregions of FLAIR hyperintensity with $\mathrm{CNI}<2$, as well as regions of normal appearing brain with elevated CNI. For the 19 patients with metabolic imaging data at all 4 scans, the changes in mean CNI2 volume from pre-RT to post-RT and from pre-RT to Fup1 were significant (from 25.2 to $16 \mathrm{~cm}^{3}, \mathrm{p}=0.0017$ and $17.2 \mathrm{~cm}^{3}, \mathrm{p}=0.0145$ respectively). The differences in spatial extent of the anatomic and metabolic lesions became even more extreme after treatment, with the overlap between the CNI2 and T2 lesion volumes being $4.6 \mathrm{~cm}^{3}$ at post-RT and $3.8 \mathrm{~cm}^{3}$ at Fup1. As can be seen from the lower bar graphs in Fig. 2, the $\operatorname{sum}(\mathrm{CNI})$, sum(CCrI), sum(nLac) and sum(nLip), which represent how abnormal the metabolite levels were within the CNI2 region from each time point relative to values in normal brain showed a similar pattern of changes.

\section{Serial results from individual patients}

Figure 3 shows an example of the serial anatomic images and overlays of CNI maps from pre-RT, post-RT, Fup1 and at two later time points prior to progression for a 47 year old patient who had received a sub-total resection, and had a KPS of 70, with PFS of 330 days and OS of 706 days. The images on the upper panel are from the post-contrast T1-weighted sequence and show that the enhancing lesion virtually disappears by post-RT (from an overall volume of $6.7 \mathrm{~cm}^{3}$ at pre-RT to $0.5 \mathrm{~cm}^{3}$ at Fup1) and a small region of new enhancement appears in the corpus callosum at the 11 month follow-up scan. The FLAIR images on the bottom panel show a large T2 lesion at pre-RT (overall volume of $55 \mathrm{~cm}^{3}$ ) with two components, one being brighter and the other being less intense. The T2 lesion was smaller at the post-RT and Fup1 scan but was beginning to impinge upon the corpus callosum at the 8 month scan and then continued expanding in the 11 month scan. The CNI2 region represented by the color overlay images in the middle panels included only a portion of the T2 lesion. The maximum CNI at pre-RT was 20.5 and at Fup1 it was 18.6, suggesting that, despite the lack of enhancement, there was substantial residual tumor. On the 8 month and 11 month scans the CNI2 lesion increased in size and had clearly crossed the corpus callosum, but was still smaller than the overall T2 lesion.

Figure 4 shows a similar time course for a second patient who was 38 years old, had a KPS of 90, a biopsy only, similar PFS of 360 days and a shorter OS of 434 days. The overall CE lesion volume at the pre-RT scan was $5.5 \mathrm{~cm}^{3}$ but reduced to $<1 \mathrm{~cm}^{3}$ on subsequent scans. The T2 lesion had an overall volume of $37 \mathrm{~cm}^{3}$ at pre-RT, reducing at the post-RT scan and 4 month scans to $25 \mathrm{~cm}^{3}$ and $20 \mathrm{~cm}^{3}$ respectively. At the 11 month scan, the T2 lesion volume was similar in size on the axial slice shown but had expanded on inferior slices. The metabolic lesion had high CNI values at all time 


\section{(a) Mean anatomic and metabolic lesion volumes $\left(\mathrm{cm}^{3}\right)$}

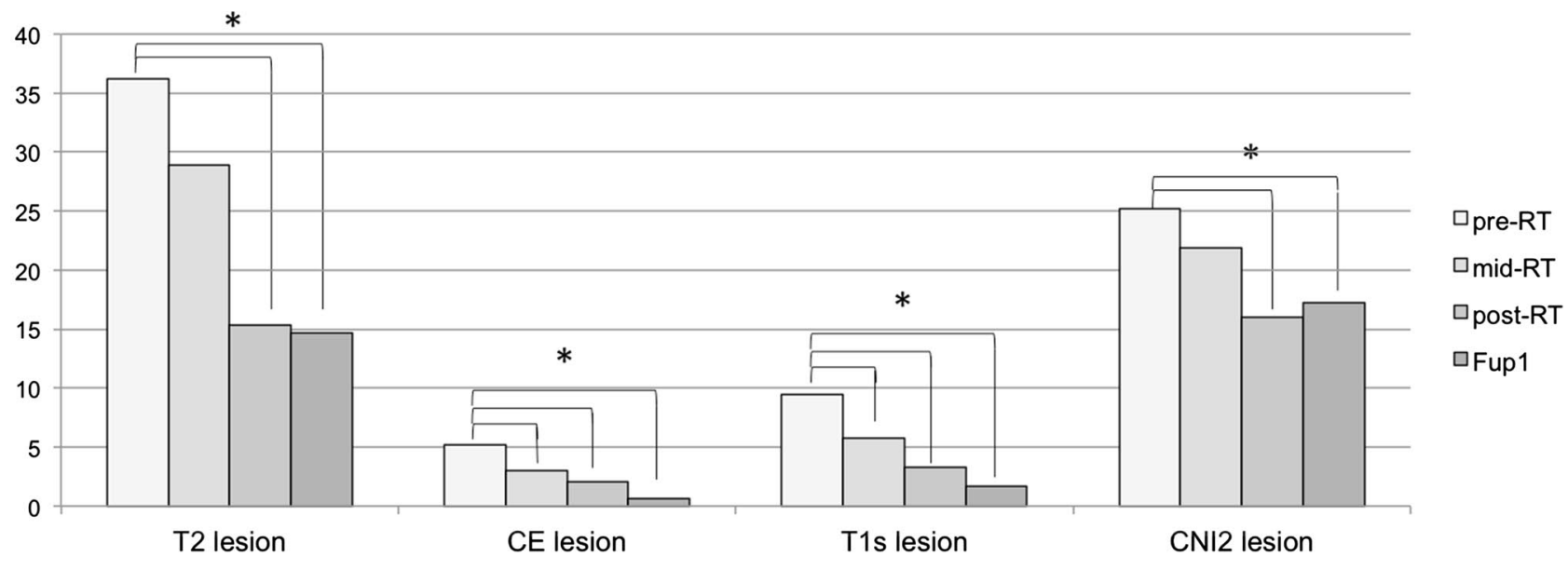

(b) Mean choline metrics in CNI2

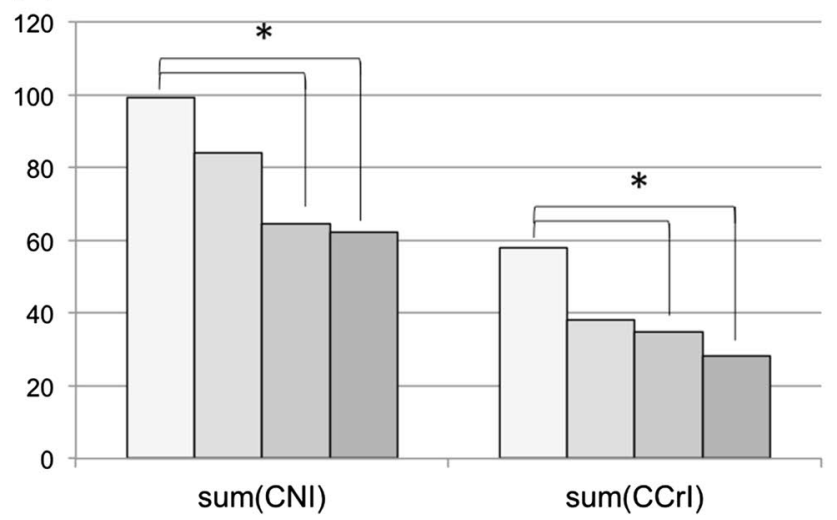

Fig. 2 Changes in mean anatomic, choline, lactate and lipid parameters for patients with scans at all four time points (pre-, mid-, post-RT and Fup1). The asterisks denote time points at which the change from pre-RT in the relevant parameter was statistically significant. The sum

points (the maximum CNI at pre-RT was 25.6 and at Fup1 was 22.9). At the initial time points, the region with $\mathrm{CNI}>2$ covered the majority of the T2 lesion, suggesting that there was substantial non-enhancing tumor. While the CNI2 lesion changed in shape during the initial 8 months, it was fairly stable in size until the 11 month scan when it became substantially larger and extended beyond the T2 lesion. There was no evidence of enhancement on the post-Gadolinium T1-weighted at this time. Once more, the metabolic data were helpful in providing confidence that the increase in the volume of the T2 lesion corresponded to infiltrative tumor. The large increase in the CNI2 lesion volume at progression was consistent with the relatively short OS.

\section{Association with survival}

The only anatomic imaging parameter that showed an association with outcome was the volume of the CEL at post-RT

\section{(c) Mean lactate/lipid metrics in $\mathrm{CNI} 2$}



values are integrals of the various parameters from voxels within the region with $\mathrm{CNI}>2$. There were 21 subjects with anatomic data and 19 subjects with metabolic data at all 4 time points

for PFS ( $p=0.029)$. In contrast, a large number of the metabolic imaging parameters considered in the analysis were associated with PFS and OS at the Fup1 (4 month) scan (see Table 1). If the population was divided into cohorts with survival either shorter or longer than the median value, it was observed that there were significant reductions in metabolite levels for both cohorts and that these changes were observed at both the post-RT and the Fup1 scans. This was true when using both the median PFS and median OS to define the cut-points.

\section{Discussion}

The results of our study highlighted the substantial changes in anatomic lesion volumes that are seen in treatments that include anti-angiogenic agents such as bevacizumab. The RANO criteria for assessing response to therapy in patients 


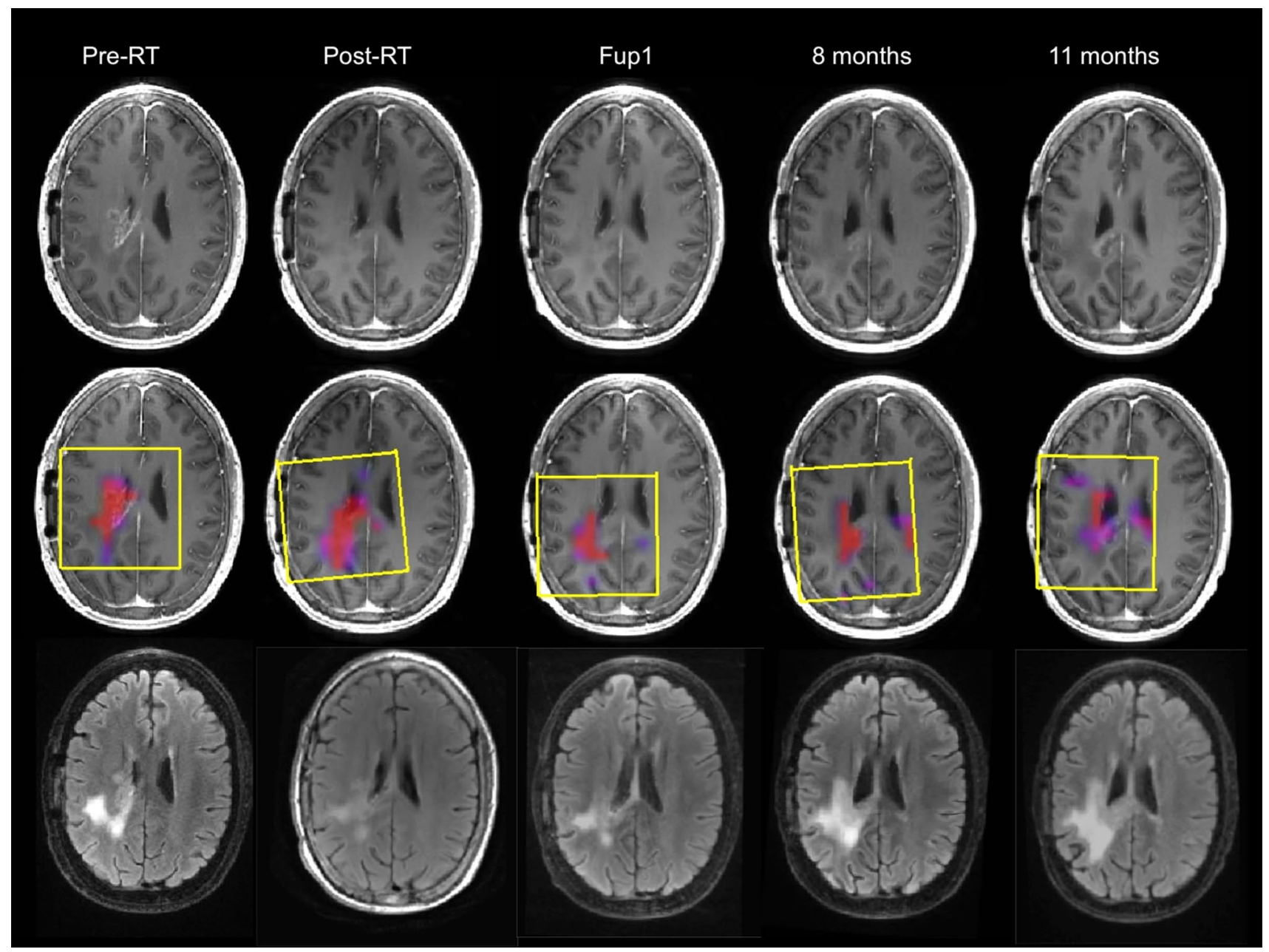

Fig. 3 Serial post-Gad T1-weighted, FLAIR images and overlaid CNI maps for a male patient who was 47 years old, had a PFS of 330 days and OS of 706 days. The yellow boxes indicating PRESS selected

with GBM depend upon changes in cross sectional diameter of the CE lesion, with secondary evaluation of the $\mathrm{T} 2$ lesion [8]. This is problematic for treatments including antiangiogenic agents because the size of the enhancing portion becomes too small for further evaluation, while the T2 lesion may include a mixture of non-enhancing tumor and non-specific treatment effects. Of interest in the current study is that the major reduction in the $\mathrm{T} 2$ lesion volume occurred between the mid- and post-RT scan, whereas the volumes of the $\mathrm{CE}$ and $\mathrm{T} 1$ subtraction lesions were significantly reduced at the mid-RT scan and continued decreasing for the next two scans. These observations are consistent with prior reports of changes in lesion volumes that are observed following treatment with anti-angiogenic agents and have been attributed to normalization of the vasculature in the tumor and associated reduction in edema [2-7]. Of interest is that the only anatomic parameter associated with PFS was the volume of the enhancing lesion at the post-RT volumes are oblique for follow-up scans because the 3D imaging and spectral data were post-processed to register them to the pre-RT exam in order to aid in making visual comparisons

scan. It is presumably a reflection of whether the lesion was sensitive and showed an immediate response to this agent.

An alternative approach that has been suggested for assessing changes in tumor burden is to use 3-D H-1 MRSI to evaluate regions with abnormal metabolism [22-24]. The $\mathrm{CNI}$ and related metrics such as the ratio of choline to NAA have previously been shown to be increased in tumor [20], and to detect areas of infiltrative tumor outside of the CE lesion [21]. This may be important for targeting radiation and other focal therapies, as well as monitoring response to treatment [22-29]. Of particular interest is our prior study of patients with newly diagnosed GBM being treated with radiation and temozolomide [12], which indicated that metabolic parameters reduced from pre- to post-RT and that values obtained from the post-RT scan were associated with overall survival.

The results of the current study indicate that there is only partial overlap between the CNI2 and T2 lesions, with there 


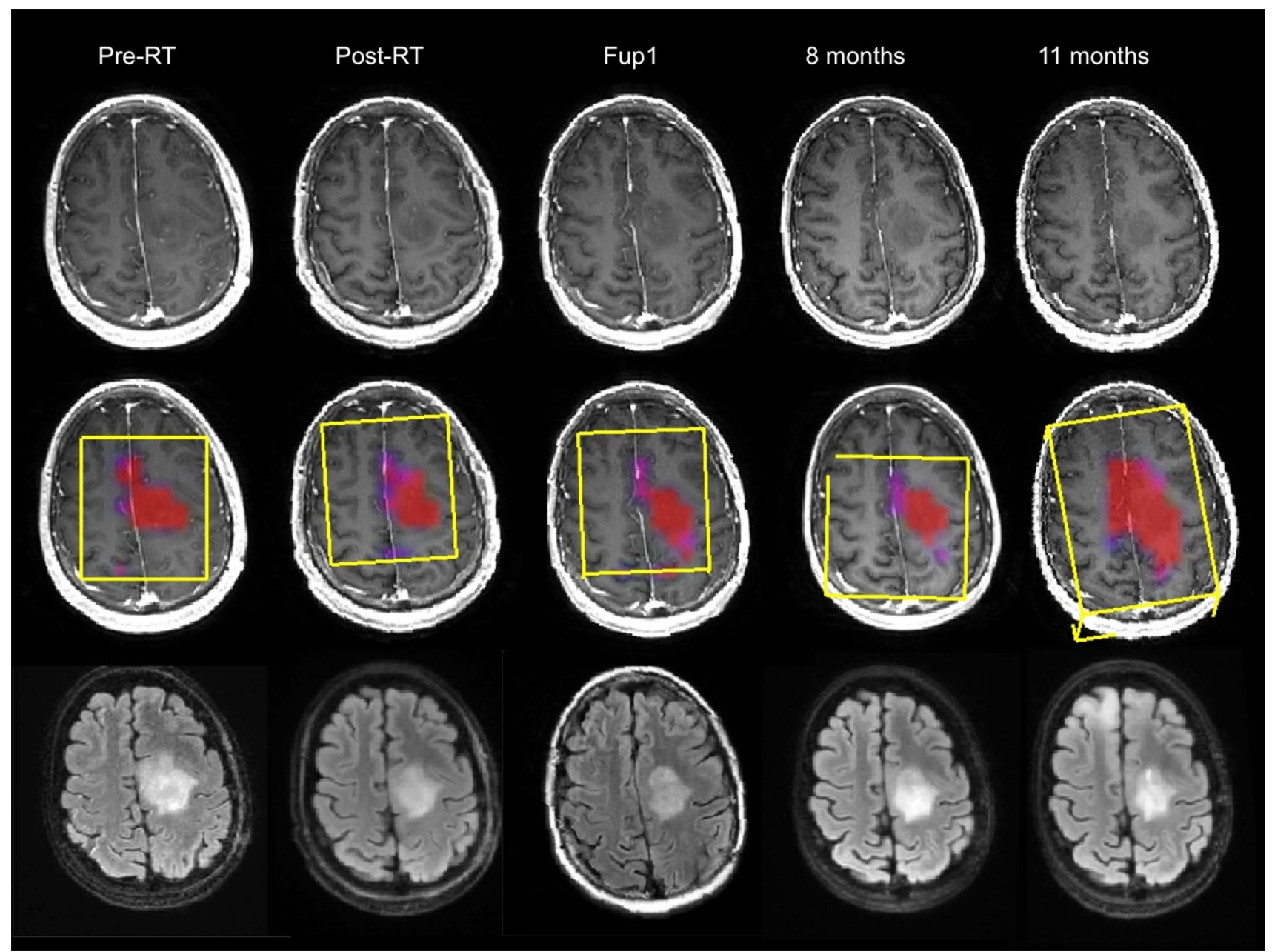

Fig. 4 Serial post-Gad T1-weighted, FLAIR images and overlaid CNI maps for a female patients who was 38 years old, had a PFS $=$ of 360 days and OS of 434 days. The yellow boxes indicating PRESS selected volumes are oblique for follow-up scans because the 3D imaging and spectral data were post-processed to register them to the pre-RT exam in order to aid in making visual comparisons
Table 1 Association of imaging and metabolic parameters with survival based upon Cox proportional hazards analysis, adjusting for age and extent of resection

\begin{tabular}{|c|c|c|c|c|c|c|c|c|}
\hline \multirow[t]{2}{*}{ Parameters } & \multicolumn{4}{|c|}{ Progression free survival } & \multicolumn{4}{|c|}{ Overall survival } \\
\hline & Pre-RT & Mid-RT & Post-RT & Fup1 & Pre-RT & Mid-RT & Post-RT & Fup1 \\
\hline \multicolumn{9}{|c|}{ Anatomic imaging } \\
\hline Volume (T2) & NS & NS & NS & NS & NS & NS & NS & NS \\
\hline Volume (CE) & NS & NS & 0.0299 & NS & NS & NS & NS & NS \\
\hline Volume (T1s) & NS & NS & NS & NS & NS & NS & NS & NS \\
\hline \multicolumn{9}{|c|}{ Metabolic imaging } \\
\hline Volume(CNI2) & NS & NS & NS & 0.0046 & NS & NS & NS & 0.0080 \\
\hline $\operatorname{Sum}(\mathrm{CNI})$ & NS & NS & NS & 0.0034 & NS & NS & NS & 0.0033 \\
\hline Sum(CCrI) & NS & NS & NS & 0.0033 & NS & NS & NS & 0.0040 \\
\hline Sum(nLac) & NS & NS & NS & 0.0019 & NS & NS & NS & 0.0010 \\
\hline Sum(nLip) & NS & NS & NS & 0.0001 & NS & NS & NS & 0.0034 \\
\hline
\end{tabular}

Metrics with $\mathrm{p}<0.05$ were considered to be significant but all of the metabolic parameters have much smaller $\mathrm{P}$ values. The number of subjects with anatomic imaging data at the each of the time points was $29,25,25$ and 27, while the number of subjects with metabolic imaging data was $28,24,21$ and 26 . There were 4 subjects censored for PFS and 10 subjects censored for OS 
being regions of FLAIR hyperintensity that are not metabolically abnormal and regions of normal appearing brain with elevated CNI values. This suggests that the spatial information provided by the metabolic data is complementary to both types of anatomic images. The color overlays of the CNI maps that are presented in the figures are helpful in relating the metabolic and anatomic data in a visual manner. A critical factor to note here is that although $9 / 31$ patients in our study were assessed based on clinical criteria as having a gross total resection, all of the subjects had regions with metabolic parameters that were consistent with tumor.

Another property of the MRSI data is that they provide measurements of levels of multiple metabolites that can be quantified and tracked with time. The reductions in parameters reflecting abnormal levels of choline relative to NAA (CNI) and creatine (CCrI) suggest that the tumor cellularity is decreasing and is therefore responding to therapy. Prior studies that used MRSI to study patients being treated with cediranib [30], radiation and temozolomide [12] and other treatment strategies $[13,31,32]$ have also showed a reduction in choline related parameters. The lower levels of lipid and lactate (nLip and nLac) within the metabolic lesion at the post-RT and the next follow-up scans indicate that the tumor is becoming better oxygenated, which is consistent with the impact of vascular normalization [21]. The associations of metabolic parameters from the follow-up scan with PFS and OS are encouraging in terms of providing surrogate measures of the effectiveness of different treatments. Including consideration of these metabolic parameters into the RANO criteria would be especially helpful for circumstances where the $\mathrm{T} 2$ lesion has increased in size and it is uncertain whether this is due to tumor progression. While the spatial resolution of MRSI is currently limited by signal to noise and acquisition times to approximately $1 \mathrm{~cm}^{3}$, the impressive results that have been obtained in this study suggest that it provides complementary information to other imaging modalities. Future clinical studies should be designed to include the acquisition of metabolic imaging data in order to determine whether these results hold for a larger cohort of patients.

\section{Conclusions}

This study supports the hypothesis that levels of choline, creatine, NAA, lactate and lipid change in response to treatment and that metrics describing these characteristics are associated with survival. Integrating 3D H-1 MRSI into the serial assessment of patients with GBM who are receiving therapies that include anti-angiogenic agents may therefore be helpful for resolving ambiguities caused by non-specific treatment effects and for predicting outcome.
Acknowledgments This research was supported by NIH Grants R01 CA127612 and P01 CA118816.

\section{Compliance with ethical standards}

Conflict of interest The authors declare that they have no conflict of interest.

Open Access This article is distributed under the terms of the Creative Commons Attribution 4.0 International License (http://creativecommons.org/licenses/by/4.0/), which permits unrestricted use, distribution, and reproduction in any medium, provided you give appropriate credit to the original author(s) and the source, provide a link to the Creative Commons license, and indicate if changes were made.

\section{References}

1. Stupp R, Hegi ME, Mason WP, van den Bent MJ, Taphoorn MJ, Janzer RC, Ludwin SK, Allgeier A, Fisher B, Belanger K, Hau P, Brandes AA, Gijtenbeek J, Marosi C, Vecht CJ, Mokhtari K, Wesseling P, Villa S, Eisenhauer E, Gorlia T, Weller M, Lacombe D, Cairncross JG, Mirimanoff RO; European Organisation for Research and Treatment of Cancer Brain Tumour and Radiation Oncology Groups; National Cancer Institute of Canada Clinical Trials Group (2009) Effects of radiotherapy with concomitant and adjuvant temozolomide versus radiotherapy alone on survival in glioblastoma in a randomised phase III study: 5-year analysis of the EORTC-NCIC trial. Lancet Oncol 10(5):459-466

2. Clarke JL, Chang S (2009) Pseudoprogression and pseudoresponse: challenges in brain tumor imaging. Curr Neurol Neurosci Rep 9:241-246

3. Pope WB, Lai A, Nghiemphu P, Mischel P, Cloughesy TF (2006) MRI in patients with high-grade gliomas treated with bevacizumab and chemotherapy. Neurology 66:1258-1260

4. Friedman HS, Prados MD, Wen PY et al (2009) Bevacizumab alone and in combination with irinotecan in recurrent glioblastoma. J Clin Oncol 27:4733-4740

5. Nghiemphu PL, Liu W, Lee Y et al (2009) Bevacizumab and chemotherapy for recurrent glioblastoma: a single-institution experience. Neurology 72:1217-1222

6. Lai A, Nghiemphu PL, Green RM et al (2009) Updated results of phase II trial of bevacizumab in combination with temozolomide and regional radiation therapy for upfront treatment of patients with newly-diagnosed glioblastoma multiforme. Neuro. Oncol 11:632

7. Gilbert MR, Dignam JJ, Armstrong TS et al A randomized trial of bevacizumab for newly diagnosed glioblastoma. N Engl J Med 2014 370:699-708

8. Wen PY, Macdonald DR, Reardon DA, Cloughesy TF, Sorensen AG, Galanis E, Degroot J, Wick W, Gilbert MR, Lassman AB, Tsien C, Mikkelsen T, Wong ET, Chamberlain MC, Stupp R, Lamborn KR, Vogelbaum MA, van den Bent MJ, Chang SM (2010) Updated response assessment criteria for high-grade gliomas: response assessment in neuro-oncology working group. J Clin Oncol 28:1963-1972

9. Nelson SJ (2011) Assessment of therapeutic response and treatment planning for brain tumors using metabolic and physiological MRI. NMR Biomed 24:734-749

10. Crawford FW, Khayal IS, McGue C et al (2009) Relationship of pre-surgery metabolic and physiological MR imaging parameters to survival for patients with untreated GBM. J Neurooncol 91(3):337-351 
11. Saraswathy S, Crawford FW, Lamborn KR et al (2009) Evaluation of MR markers that predict survival in patients with newly diagnosed GBM prior to adjuvant therapy. J Neurooncol 91(1):69-81

12. Li Y, Lupo JM, Parvataneni R, Lamborn KR, Cha S, Chang SM, Nelson SJ (2013) Survival analysis in patients with newly diagnosed glioblastoma using pre- and post-radiotherapy MR spectroscopic imaging. Neuro. Oncol 15(5):607-617

13. Clarke JL, Molinaro AM, Phillips JJ, Butowski NA, Chang SM, Perry A, Costello JF, DeSilva AA, Rabbitt JE, Prados MD (2014) A single-institution phase II trial of radiation, temozolomide, erlotinib, and bevacizumab for initial treatment of glioblastoma. Neuro. Oncol 16(7):984-990

14. Park I, Chen AP, Zierhut ML et al (2011) Implementation of 3 T lactate-edited 3D $1 \mathrm{H}$ MR spectroscopic imaging with flyback echo-planar readout for glioma patients. Ann Biomed Eng 39:193-204

15. Studholme C, Hill D, Hawkes D (1999) An overlap invariant entropy measure of 3D medical image alignment. Pattern Recog 32:71-86

16. Ellingson BM, Kim HJ, Woodworth DC, Pope WB, Cloughesy JN, Harris RJ, Lai A, Nghiemphu PL, Cloughesy TF (2014) Recurrent glioblastoma treated with bevacizumab: contrastenhanced T1-weighted subtraction maps improve tumor delineation and aid prediction of survival in a multicenter clinical trial. Radiology 271(1):200-210

17. Nelson SJ (2001) Analysis of volume MRI and MR spectroscopic imaging data for the evaluation of patients with brain tumors. Magn Reson Med 46(2):228-239

18. Crane JC, Olson MP, Nelson SJ (2013) SIVIC: open-source, standards-based software for dicom $\mathrm{mr}$ spectroscopy workflows. Int J Biomed Imaging 2013:169526

19. McKnight TR, Noworolski SM, Vigneron DB, Nelson SJ (2001) An automated technique for the quantitative assessment of 3D-MRSI data from patients with glioma. J Magn Reson Imaging 13(2):167-177

20. McKnight TR, von dem BMH, Vigneron DB et al (2002) Histopathological validation of a three-dimensional magnetic resonance spectroscopy index as a predictor of tumor presence. $\mathrm{J}$ Neurosurg 97(4):794-802

21. Muruganandham M, Clerkin PP, Smith BJ, Anderson CM, Morris A, Capizzano AA, Magnotta V, McGuire SM, Smith MC, Bayouth JE, Buatti JM (2014) 3-Dimensional magnetic resonance spectroscopic imaging at $3 \mathrm{~T}$ for early response assessment of glioblastoma patients during external beam radiation therapy. Int J Radiat Oncol Biol Phys 90(1):181-189

22. Lu-Emerson C, Duda DG, Emblem KE, Taylor JW, Gerstner ER, Loeffler JS, Batchelor TT, Jain RK (2015) Lessons from antivascular endothelial growth factor and anti-vascular endothelial growth factor receptor trials in patients with glioblastoma J Clin Oncol 33(10):1197-1213
23. Laprie A, Catalaa I, Cassol E, McKnight TR, Berchery D, Marre D, Bachaud JM, Berry I, Moyal EC (2008) Proton magnetic resonance spectroscopic imaging in newly diagnosed glioblastoma: predictive value for the site of postradiotherapy relapse in a prospective longitudinal study. Int J Radiat Oncol Biol Phys 70(3):773-781

24. Lichy MP, Bachert P, Hamprecht F, Weber MA, Debus J, SchulzErtner D, Schlemmer HP, Kauczor HU (2006) Application of (1) $\mathrm{H}$ MR spectroscopic imaging in radiation oncology: choline as a marker for determining the relative probability of tumor progression after radiation of glial brain tumors. Rofo 178(6):627-633

25. Pirzkall A, Li X, Oh J, Chang S, Berger MS, Larson DA, Verhey LJ, Dillon WP, Nelson SJ (2004) 3D MRSI for resected high-grade gliomas before RT: tumor extent according to metabolic activity in relation to MRI. Int J Radiat Oncol Biol Phys 59(1):126-137

26. Guo J, Yao C, Chen H, Zhuang D, Tang W, Ren G, Wang Y, Wu J, Huang F, Zhou L (2012) The relationship between Cho/NAA and glioma metabolism: implementation for margin delineation of cerebral gliomas. Acta Neurochir (Wien) 154(8):1361-1370

27. Shim H, Wei L, Holder CA, Guo Y, Hu XP, Miller AH, Olson JJ (2014) Use of high-resolution volumetric MR spectroscopic imaging in assessing treatment response of glioblastoma to a HDAC inhibitor. AJR Am J Roentgenol 203(2):W158-W165

28. Stadlbauer A, Buchfelder M, Doelken MT, Hammen T, Ganslandt O (2011) Magnetic resonance spectroscopic imaging for visualization of the infiltration zone of glioma. Cent Eur Neurosurg 72(2):63-69

29. Hattingen E, Jurcoane A, Bähr O, Rieger J, Magerkurth J, Anti S, Steinbach JP, Pilatus U (2011) Bevacizumab impairs oxidative energy metabolism and shows antitumoral effects in recurrent glioblastomas: a 31P/1 H MRSI and quantitative magnetic resonance imaging study. Neuro. Oncol 13(12):1349-1363

30. Kim H, Catana C, Ratai EM, Andronesi OC, Jennings DL, Batchelor TT, Jain RK, Sorensen AG (2011) Serial magnetic resonance spectroscopy reveals a direct metabolic effect of cediranib in glioblastoma. Cancer Res. 71(11):3745-3752.

31. Pirzkall A, McGue C, Saraswathy S, Cha S, Liu R, Vandenberg S, Lamborn KR, Berger MS, Chang SM, Nelson SJ (2009) Tumor regrowth between surgery and initiation of adjuvant therapy in patients with newly diagnosed glioblastoma. Neuro Oncol 11(6):842-852

32. Park I, Tamai G, Lee MC, Chuang CF, Chang SM, Berger MS, Nelson SJ, Pirzkall A (2007) Patterns of recurrence analysis in newly diagnosed GBM following 3D conformal radiation therapy with respect to pre-RT MR spectroscopic findings. Int J Radiat Oncol Biol Phys 69(2):381-389 